\title{
Influence of Selective Serotonin Reuptake Inhibitors on Bleeding Risk in Patients with Severe Thrombocytopenia after Chemotherapy: A Retrospective Study
}

\author{
Claudia Schäfer Alix O'Meara Dimitrios A. Tsakiris Michael Medinger \\ Jakob R. Passweg Martin Stern
}

Division of Hematology, University Hospital Basel, Basel, Switzerland

Several in vivo and in vitro studies have indicated that selective serotonin reuptake inhibitors (SSRIs) may increase the risk of bleeding [1-6]. The highest risk of hemostatic complications has been documented for patients treated concurrently with drugs interfering with plasmatic hemostasis or with platelet function (e.g. oral anticoagulation and nonsteroidal anti-inflammatory drugs) and for gastrointestinal bleeding as an endpoint. In contrast, other studies have failed to detect an association between SSRI treatment and a higher risk of bleeding [7-9].

Clinically relevant bleeding due to iatrogenic thrombocytopenia is one of the main complications of highdose chemotherapy used in the treatment of leukemia [10]. To date, no studies have analyzed whether administration of SSRIs is safe in terms of bleeding risk in such a population at a highly elevated risk for bleeding [11].

To assess the bleeding risk, we analyzed a cohort of 300 patients with severe iatrogenic thrombocytopenia undergoing high-dose chemotherapy for acute or chronic leukemia ( $\mathrm{n}=100$ ) or myeloablative conditioning chemotherapy followed by autologous $(\mathrm{n}=100)$ or allogeneic $(n=100)$ hematopoietic stem cell transplantation (HSCT). Data were collected from charts of consecutive patients treated at the Division of Hematology at Basel University Hospital. Data were analyzed from the start
(C) 2014 S. Karger AG, Basel

0001-5792/14/1333-0317\$39.50/0 of chemotherapy, or in case of HSCT from the day of the allogeneic or autologous HSCT until discharge. During treatment, clinical examination and laboratory tests were performed every day. For bleeding prophylaxis, irradiated thrombocyte concentrates collected by single donor apheresis were given at a thrombocyte count of $<30 \times 10^{9} / 1$ when treated with low-molecular-weight heparin $(\mathrm{LMWH}),<20 \times 10^{9} / \mathrm{l}$ in patients with fever or graft-versus-host disease, or when the morning platelet count was $<10 \times 10^{9} / 1$. Patients with a developing thrombosis received LMWH as a 24 -hour infusion with a target anti-FXa level of $0.2-0.4 \mathrm{IE} / \mathrm{ml}$ (measured daily), and LMWH infusion was immediately stopped when a bleeding event scored $\geq 3$ occurred. Veno-occlusive disease prophylaxis with unfractionated heparin was given to patients treated with allogeneic HSCT after myeloablative conditioning with a dose of 5,000 units per day as a continuous infusion until engraftment. All protocols were approved by the ethics committee and all patients gave informed consent to their treatment and to having their data analyzed.

Antidepressant drugs were categorized as SSRIs or non-SSRIs. The dose of antidepressants administered was not considered. The bleeding events were graduated using the ITP bleeding score from 0 (no hemorrhage), 1 
Fig. 1. a Distribution of severity of bleeding events $(n=187)$ occurring in 123 patients with thrombocytopenia after intensive chemotherapy. Severity is graded according to the ITP bleeding score: 1 (minor bleeding, e.g. few petechiae), 2 (mild bleeding with many petechiae and bruises, but without mucosal bleeding), 3 (moderate bleeding including mucosal, not requiring intervention), 4 (severe bleeding including internal hemorrhage, requiring intervention), and 5 (life-threatening or fatal bleeding). b Cumulative incidences (CI) of grade 1-5 (any grade) and grade 3-5 (moderate to severe) bleeding events.

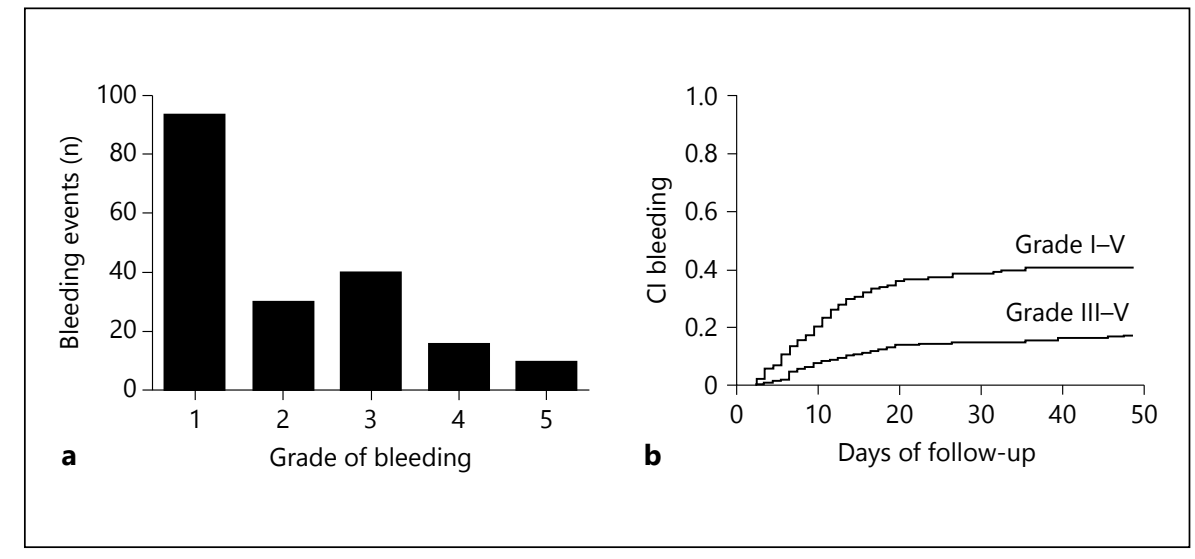

(minor bleeding, e.g. few petechiae), 2 (mild bleeding with many petechiae and bruises, but without mucosal bleeding), 3 (moderate bleeding including mucosal, not requiring intervention), 4 (severe bleeding including internal hemorrhage, requiring intervention), and 5 (lifethreatening or fatal bleeding) [12].

The effect of SSRI administration on bleeding risk was estimated using incidence rates (bleeding events per inhospital day undergoing or not undergoing SSRI/nonSSRI treatment) and multivariable Cox models. Incidence rates were compared using Pearson's $\chi^{2}$ test. In Cox models, SSRI and non-SSRI use was modeled as a timedependent covariable, i.e. the effect of drug administration was estimated in patients on treatment versus those not receiving SSRIs or non-SSRIs. Estimates were adjusted for factors known to influence the bleeding risk: thrombocyte count, inflammation (modeled by C-reactive protein), azotemia (modeled by blood urea nitrogen), treatment with LMWH, and the presence of graft-versushost disease after allogeneic HSCT [11]. All covariables were measured or graded daily during hospitalization and considered in the analysis. Non-time-dependent factors such as type of treatment (chemotherapy vs. autologous vs. allogeneic HSCT) were also included. All p values were 2 -sided and were considered significant if $<0.05$. All statistical analyses were performed using STATA 12 (StataCorp LP, College Station, Tex., USA).

In our cohort, the median age was 50 years (range: $18-$ 80). 158 patients were treated for acute myeloid leukemia or myelodysplastic syndrome, 23 for acute lymphoblastic leukemia, 56 for multiple myeloma, 40 for lymphoproliferative disorders, 9 for chronic myeloid leukemia, and 14 for other diseases. Median duration of in-hospital treatment was 24 days (range: 5-66) for a total of 7,521 patient days. The majority of patients $(256 / 300,85 \%)$ received one or more platelet transfusions. Of the 300 patients, 75 (25\%) were treated with an antidepressant (SSRIs $n=43$, non-SSRIs $n=22$, or both $n=10$ ). The mean duration of antidepressant treatment during the inpatient period in this study was 16 days (range: 2-39) for SSRIs and 23 days (range: 3-66) for non-SSRIs. On a total of 834 patient days ( $11 \%$ of all days), patients were under treatment with an SSRI, and on 687 patient days (9\% of all days), patients were under treatment with a non-SSRI. In the SSRI group, the administered drugs were (es)citalopram (given to 48 patients) and sertraline (given to 5 patients). From the non-SSRI group, mirtazapine was given to 17 patients, venlaflaxine to 8 patients, trimipramine to 6 patients, and the remaining antidepressants once (amitriptyline, clomipramine, mianserin, and trazodone).

Bleeding events of a lower degree such as petechiae occur frequently, but without a major impact on prognosis. We therefore analyzed all grades of bleeding (grades 1-5), as well as the more clinically meaningful moderate to severe bleeding events (grades 3-5). In this patient population, a total of 187 bleeding events were recorded in 123 patients ( 93 grade 1, 30 grade 2, 40 grade 3, 15 grade 4, 9 grade 5 ; fig. 1a). The cumulative incidences of grade 1-5 and grade $3-5$ bleeding at day 50 after the start of treatment were $41 \pm 3 \%$ and $17 \pm 2 \%$, respectively (fig. $1 \mathrm{~b}$ ). Twenty-three bleeding events ( $12 \%$ of all events: 13 grade 1,5 grade 2, 3 grade 3, 2 grade 4 , and 0 grade 5) occurred during SSRI treatment. Twenty bleeding events (11\% of all events: 10 grade 1, 4 grade 2, 3 grade 3,3 grade 4 , and 0 grade 5) occurred during non-SSRI treatment. Crude incidence rates per day of in-hospital days showed no increased rate of bleeding of any grade in patients on treatment with SSRIs or non-SSRIs, when compared to patients not undergoing such treatment (fig. 2). Importantly, no bleeding episodes of grade 5 (life-threatening or 


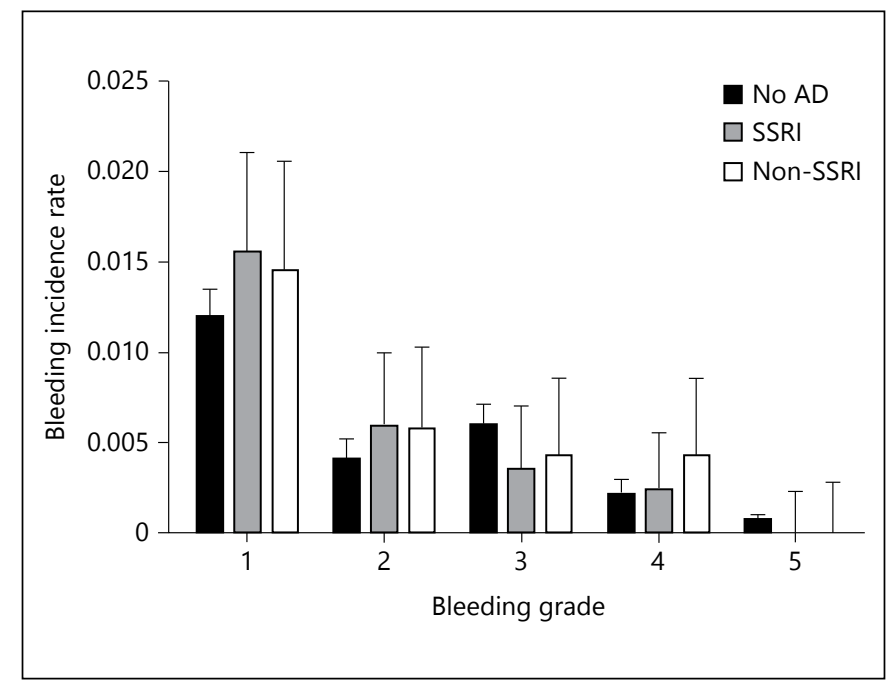

Fig. 2. Incidence rates (per day of in-hospital treatment) of bleeding events graded by the ITP bleeding score in patients not undergoing antidepressant treatment (no AD), and in patients with SSRI or non-SSRI treatment.

fatal) were noted in patients under SSRI or non-SSRI treatment.

In multivariable adjusted Cox regression analyses, we found no elevated risk for bleeding in patients undergoing SSRI or non-SSRI treatment, either for bleeding of any grade or for bleeding of grades 3-5 [hazard ratio (HR) for SSRIs for bleeding grade 1-5: 0.98, 95\% CI: 0.63-1.37, $\mathrm{p}=0.92$; HR for SSRIs for grade $3-5$ bleeding: $0.48,95 \%$ CI: $0.17-1.37, \mathrm{p}=0.17$; HR for non-SSRIs for bleeding grade 1-5: 0.94, 95\% CI: 0.64-1.40, $\mathrm{p}=0.77$; HR for nonSSRIs for grade $3-5$ bleeding: $1.11,95 \%$ CI: $0.52-2.36, \mathrm{p}=$ 0.78]. Interaction analysis assessed if the HRs for SSRIs or non-SSRIs regarding bleeding risk were modulated by platelet counts, and found no significant interaction ( $\mathrm{p}=$ 0.72 for SSRIs, $\mathrm{p}=0.88$ for non-SSRIs), suggesting that both types of antidepressants do not increase the risk for bleeding even in patients with very low platelet counts. Similarly, no significant interaction on bleeding risk was found in patients treated with an SSRI or a non-SSRI and concurrent LMWH application ( $\mathrm{p}=0.97$ and $\mathrm{p}=0.94$, respectively). Thirty bleeding events (16\% of all events) occurred in the gastrointestinal tract. Similar to the overall risk, neither SSRI nor non-SSRI treatment significantly increased the risk for gastrointestinal bleeding (HR for SSRIs: $0.97,95 \%$ CI: $0.28-3.30, \mathrm{p}=0.96$; HR for nonSSRIs: 1.48 , 95\% CI: 0.67-3.23, $\mathrm{p}=0.33$ ).

In our retrospective study, we found no significant influence of SSRI treatment on the risk of bleeding, either of any grade or of moderate to severe grade. Subgroup analyses suggested that SSRI/non-SSRI treatment also does not increase the risk for gastrointestinal bleeding, and does not increase the bleeding risk in patients with severe thrombocytopenia.

In previous studies, drugs with the strongest affinity to the 5HT receptor, i.e. fluoxetine, paroxetine, and sertraline, were most frequently associated with abnormal bleeding [13]. Possibly, the moderate affinity for the transporter of most antidepressants used in this cohort might explain the lack of influence on bleeding risk in this study. Another potential mitigating factor is platelet transfusion, which was administered to the majority of patients in this study. Healthy donor platelets are rich in serotonin, and it was not investigated whether (and if yes after how much time) these transfused platelets are functionally compromised when given to patients treated with serotonin reuptake inhibitors.

Apart from the effect on serotonin in platelets, other mechanisms responsible for increased bleeding risk in patients on SSRI treatment have been discussed [14]. For example, the increased gastrointestinal bleeding risk may be caused by enhanced gastric acid secretion (due to SSRI use) rather than the influence of thrombocyte aggregation. In our population, prophylaxis with the proton pump inhibitor pantoprazole was administered to all patients to reduce gastric acid production, which may have also mitigated effects that SSRI may have on gastrointestinal bleeding risk. A limitation of this study was that the in-hospital exposure to antidepressant therapy was relatively short, and no data were available on the duration of treatment in patients already treated at the start of hospitalization. However, while earlier studies have suggested that serotonin reuptake inhibition would only increase bleeding risk after several weeks of treatment, more recent studies have shown that an increased risk for gastrointestinal bleeding is already apparent after 1 week of SSRI treatment [15].

In conclusion, we could not detect an effect of SSRI or non-SSRI treatment on bleeding events in patients with severe thrombocytopenia induced by chemotherapy. SSRIs [e.g. (es)citalopram] and non-SSRIs (such as mirtazapine) may be safely used in patients with severe thrombocytopenia.

\section{Acknowledgements}

This study was supported by grants from the Swiss National Science Foundation (PP00P3_128461/1) and the Olga Mayenfisch Stiftung (both to M.S.). 


\section{References}

$>1$ Halperin D, Reber G: Influence of antidepressants on hemostasis. Dialogues Clin Neurosci 2007;9:47-59.

$>2$ Scharf RE: Drugs that affect platelet function. Semin Thromb Hemost 2012;38:865-883.

$\checkmark 3$ Butler J, Leonard BE: The platelet serotonergic system in depression and following sertraline treatment. Int Clin Psychopharmacol 1988;3:343-347.

-4 Helmeste DM, Tang SW, Reist C, Vu R: Serotonin uptake inhibitors modulate intracellular Ca2 + mobilization in platelets. Eur J Pharmacol 1995;288:373-377.

$>5$ Hergovich N, Aigner M, Eichler HG, Entlicher J, Drucker C, Jilma B: Paroxetine decreases platelet serotonin storage and platelet function in human beings. Clin Pharmacol Ther 2000;68:435-442.

$\checkmark 6$ Hallback I, Hagg S, Eriksson AC, Whiss PA: In vitro effects of serotonin and noradrenaline reuptake inhibitors on human platelet adhesion and coagulation. Pharmacol Rep 2012; 64:979-983.
7 Opatrny L, Delaney JA, Suissa S: Gastro-intestinal haemorrhage risks of selective serotonin receptor antagonist therapy: a new look. Br J Clin Pharmacol 2008;66:76-81.

$>8$ Mort JR, Aparasu RR, Baer RK: Interaction between selective serotonin reuptake inhibitors and nonsteroidal antiinflammatory drugs: review of the literature. Pharmacotherapy $2006 ; 26: 1307-1313$.

$\checkmark 9$ Wallerstedt SM, Gleerup H, Sundstrom A, Stigendal L, Ny L: Risk of clinically relevant bleeding in warfarin-treated patients - influence of SSRI treatment. Pharmacoepidemiol Drug Saf 2009; 18:412-416.

10 de Abajo FJ, Jick H, Derby L, Jick S, Schmitz $\mathrm{S}$ : Intracranial haemorrhage and use of selective serotonin reuptake inhibitors. Br J Clin Pharmacol 2000;50:43-47.
1 Pihusch M: Bleeding complications after hematopoietic stem cell transplantation. Semin Hematol 2004;41:93-100.

12 Buchanan GR, Adix L: Grading of hemorrhage in children with idiopathic thrombocytopenic purpura. J Pediatr 2002;141:683-688.

13 Meijer WE, Heerdink ER, Nolen WA, Herings RM, Leufkens HG, Egberts AC: Association of risk of abnormal bleeding with degree of serotonin reuptake inhibition by antidepressants. Arch Intern Med 2004;164:23672370.

14 Andrade C, Sandarsh S, Chethan KB, Nagesh KS: Serotonin reuptake inhibitor antidepressants and abnormal bleeding: a review for clinicians and a reconsideration of mechanisms. J Clin Psychiatry 2010;71:1565-1575.

15 Wang YP, Chen YT, Tsai CF, Li SY, Luo JC, Wang SJ, Tang CH, Liu CJ, Lin HC, Lee FY, Chang FY, Lu CL: Short-term use of serotonin reuptake inhibitors and risk of upper gastrointestinal bleeding. Am J Psychiatry 2014;171: 54-61. 